\title{
A systematic review and meta-analysis on the efficacy and safety of traditional Chinese medicine bath in the treatment of psoriasis vulgaris
}

\author{
Hua Lei ${ }^{1,2 \#}$, Minghui Chen ${ }^{1,2 \#}$, Nan Zhang ${ }^{1,2}$, Xiaojuan Guo ${ }^{1,2}$, Xiaohui Yuan ${ }^{1,2}$, Lina Tang ${ }^{1,2}$, \\ Chuanpeng Ying ${ }^{1,2}$, Jun $\mathrm{Xie}^{1,2}$
}

${ }^{1}$ Institute of Dermatology and Venereology, Sichuan Provincial People's Hospital, University of Electronic Science and Technology of China, Chengdu, China; ${ }^{2}$ Chinese Academy of Sciences Sichuan Translational Medicine Research Hospital, Chengdu, China

Contributions: (I) Conception and design: H Lei, M Chen, C Ying, J Xie; (II) Administrative support: N Zhang, X Guo; (III) Provision of study materials or patients: H Lei, M Chen, X Guo, X Yuan, L Tang; (IV) Collection and assembly of data: All authors; (V) Data analysis and interpretation: H Lei, N Zhang, C Ying, J Xie; (VI) Manuscript writing: All authors; (VII) Final approval of manuscript: All authors.

\#These authors contributed equally to this work.

Correspondence to: Jun Xie; Chuanpeng Ying. Institute of Dermatology and Venereology, Sichuan Academy of Medical Sciences and Sichuan Provincial People's Hospital, 32 West Section 2, First Ring Road, Qingyang District, Chengdu 610072, China. Email: xiejun790420@126.com; yingchuanpeng@163.com.

Background: To clarify the efficacy and safety of traditional Chinese medicine (TCM) bath in the treatment of psoriasis vulgaris, meta-analysis and systematic evaluation were adopted to comprehensively evaluate the published articles.

Methods: Combing the terms "traditional Chinese medicine bath" and "psoriasis vulgaris", the articles were searched for in the databases of China Knowledge Network (CNKI), Baidu scholar, Wanfang, Chinese Biomedical Literature Database (CBM), Weipu Database, Medline, Embase, Chinese Medical Citation Index (CMCI), and PubMed. The quality of articles was evaluated using the RevMan 5.3 software provided by the Cochrane system.

Results: A total of 13 articles were included. From the funnel chart drawn, it could be seen that the circles and the midline of most articles were not symmetrical, and the publication was biased, so the conclusions obtained were relatively credible. The heterogeneity analysis of TCM bath in treatment of psoriasis vulgaris showed that 13 articles reporting the total effective rate (TER) and the TERs of the two groups displayed obvious differences [ $\mathrm{P}<0.05,95 \%$ confidence interval (CI): 2.9 to 4.77]; there were 8 articles reporting the incidence of adverse reactions, and the incidence of adverse reactions between the two groups was greatly different $(\mathrm{P}<0.05,95 \% \mathrm{CI}: 0.22$ to 0.77$)$; and there were 9 articles reporting the psoriasis area and severity index, and the overall efficacy was greatly different between the two groups ( $\mathrm{P}<0.05,95 \% \mathrm{CI}:-5.38$ to -1.88 ). Discussion: The TER of the meta-analysis of treatment in this study was higher in the experimental group; the incidence of adverse reactions was lower in the experimental group; and the area and severity of psoriasis were lower in the experimental group, so it was concluded that the TCM bath had a significant effect on the treatment of psoriasis vulgaris.

Keywords: Traditional Chinese medicine bath (TCM bath); meta-analysis; psoriasis vulgaris

Submitted Aug 08, 2021. Accepted for publication Sep 24, 2021.

doi: 10.21037/apm-21-2386

View this article at: https://dx.doi.org/10.21037/apm-21-2386 


\section{Introduction}

As the largest organ of the human body, the skin wraps the body in direct contact with the outside world, protects, regulates temperature, and processes sensory stimuli. There are many kinds of skin diseases. Psoriasis is a chronic inflammatory skin disease that is prone to relapse (1) and its characteristic damage is red papules, with silvery white scales and flushing membranous basal layer after scratching, and spotting bleeding after scratching. White scales, shiny films, and spotting bleeding are the triad of psoriasis diagnosis (2). It is very common in young people, and the incidence rate in men is higher than that in women. It is more common in the north than in the south of China, is easily aggravated during spring and winter (3), and relieved in summer and autumn. Some internationally- and China-based studies have not yet fully clarified the etiology and pathogenesis of psoriasis, and its pathogenesis may be related to genetic factors and abnormal immune function (4). Psoriasis is clinically divided into four types: pustular, erythrodermic, vulgaris, and arthropathic. Among them, psoriasis vulgaris is the most common (5), has slower complications, and is more likely to relapse after healing. The skin lesions of psoriasis vulgaris can be roughly divided into three phases: progressive phase, stationary phase, and degenerative phase (6). It can be beneficial to adhere to strict environmental hygiene, exercise frequently, and actively reduce predisposing factors such as excessively spicy food, thereby improving immunity and reducing the disease recurrence (7).

Medicated soaking is one of the external treatment methods in traditional Chinese medicine (TCM) (8). The earliest medical soak prescription comes from China's Prescriptions for Fifty-two Diseases, which is for the treatment of infant epilepsy. A TCM bath involves the immersion of certain parts of the body or the whole body in a water-filled vessel (9), using the water temperature to stimulate the skin, meridians, and acupoints to promote transdermal absorption and achieve the therapeutic treatment effect (10). It acts directly on the skin without gastrointestinal destruction, has less toxicity and side effects than oral medicine, and places less burden on the liver. Medicated bath is divided into local medicated baths and systemic medicated baths (11). Local medicated baths are mostly used for soaking the feet and calves; whole body medicated baths are used for soaking and fumigating other parts of the body except the head and neck (12), and are generally used for diseases affecting wider areas. A related study (13) showed that drug ions in drug bath solution entered the body through the absorption, diffusion, and radiation of the skin and mucosa, to avoid the first-pass effect and increase the drug concentration. At the same time, damp-heat promotes local vasodilation (14), and promotes blood and lymphatic circulation, thus leading to the cure of the disease (15). The patient should be advised to rise slowly after their medicated bath to avoid orthostatic hypotension. Medicated baths should not be taken half an hour after meals or before meals. The duration of medicated bath should not be too long (16), and a whole body medicated bath should not be performed before going to bed. The same medicated bath medicine can be used repeatedly, and 1 dose of medicine can be used for 5-7 days in winter and 2-3 days in summer.

At present, there are a large number of reports on the treatment of psoriasis vulgaris with TCM bath in China and foreign countries, but the sample size of most literatures is less, the sample types are mostly similar, and the regional age distribution is more concentrated, so the research results are not universal (17). Therefore, in order to further improve the theoretical guidance of Chinese medicine bath in treating psoriasis, meta-analysis was applied in this study, which mainly aimed at the reference literatures published since the establishment of the database on the treatment of psoriasis vulgaris with Chinese medicine bath, and comprehensively analyzed its safety and effectiveness, so as to provide reliable guidance for the future clinical treatment of psoriasis vulgaris. We present the following article in accordance with the PRISMA reporting checklist (available at https://dx.doi.org/10.21037/apm-21-2386).

\section{Methods}

Participants in the experimental group were treated with TCM bath and ultraviolet radiation. The dosage, time, interval, treatment process, and properties of ultraviolet radiation were not limited. Participants in the control group received a single dose of ultraviolet radiation, and the treatment time, exposure intensity, treatment interval, and ultraviolet radiation properties were not limited.

\section{Inclusion and exclusion criteria of the articles}

The inclusion criteria were defined as follows: articles which were randomized controlled trial (RCT); articles including patients clinically confirmed as psoriasis vulgaris without other skin disease history as participants; articles with pathological control analysis with a reliable index comparison in the $95 \%$ confidence interval (CI); and articles involving patients with TCM bath treatment of psoriasis as 
the experimental group.

The exclusion criteria were as follows: articles including elderly and children as participants; articles which were meta-analysis on relevant conference speeches and review literature; articles taking animals as participants; articles with unavailable complete data; articles researching other types of psoriasis; and articles researching other diseases of the circulatory system, such as hypertension.

The titles, abstracts, and full texts of the articles were independently screened by two senior experts and three preliminary operations were performed before the final screening. Any inconsistencies between the two experts were resolved by discussion or arbitration with a third expert.

\section{Article retrieval}

The articles were searched for in the databases of China Knowledge Network (CNKI), Baidu scholar, Wanfang, Chinese Biomedical Literature Database (CBM), Weipu Database, Medline, Embase, Chinese Medical Citation Index (CMCI), and PubMed. The articles on TCM bath in treatment of psoriasis vulgaris published from the establishment of the database to 1 June 2021 were searched for and screened using compound logic search Boolean logic search method. The Chinese and English databases were searched using "TCM bath" and "psoriasis vulgaris" as terms. The quality of the articles was evaluated based on the RevMan 5.3 software (Review Manager; Copenhagen: The Nordic Cochrane Center, The Cochrane Collaboration, 2014) provided by the Cochrane system. The above two search terms were combined to determine the included references after searching, and then traced using the search engine. Relevant experts and researchers in the field were contacted to obtain the most up-to-date research progress.

\section{Data extraction}

The data were extracted by two experts independently using Microsoft Excel, and three pre-experiments were performed before data extraction. A consistent conclusion was obtained through detailed reading and discussion. The extracted data included the following content: title of the article, name of the first author, publication time; name of the publication; the type of research design; and the sample sizes of the experimental group and the control group participants. In addition, the total effective rate (TER), incidence of adverse reactions, affected area, and severity index were extracted.

\section{Risk of bias assessment and quality assessment}

The risk of bias was assessed by two researchers independently. Any inconsistencies between the two experts were resolved by discussion or arbitration with a third expert. In this study, the recommendations of the National Institute of Clinical Excellence (NICE) for quality evaluation were applied. The specific items of the quality evaluation are shown in Table 1. The score for each item was 1 point, and the score range was 0 to 8 points. A score of 6-8 was indicated as high-quality research, 3-6 referred to medium-quality research, and 0-2 indicated low-quality research. The quality evaluation was conducted by two researchers separately, and the consistency evaluation was obtained through discussion when the opinions were inconsistent. The above items were judged as "high-risk bias", "low-risk bias", and "unclear risk", respectively.

\section{Observation indicators}

The outcome indicators mainly depended on the TER of clinical treatment, incidence of adverse reactions, psoriasis area, and psoriasis severity index.

\section{Statistical analysis}

The software Stata SE12.0 (StataCorp., College Station, TX, USA) was used for statistical analysis. The bias risk assessment chart was drawn using the RevMan 5.3 software. Each effect was expressed using a 95\% CI. When $\mathrm{P}>0.1$ and $\mathrm{I}^{2} \leq 50 \%$, the fixed effects model (FEM) was used for meta-analysis. When $\mathrm{P}<0.1$ and $\mathrm{I}^{2}>50 \%$, the random effects model (REM) was used for meta-analysis.

\section{Results}

\section{Search results and basic characteristics of the included articles}

A total of 526 documents were obtained in this search, of which 300 were eliminated by titles and abstracts, and 213 were eliminated after reading the full text. Finally, 13 documents were obtained for meta-analysis. The following Figure 1 is the flow chart of literature retrieval. The main reasons for the deletion of literatures are: 100 duplicate literatures, 35 literatures whose research type is not case randomized controlled analysis, 47 literatures whose research object is not psoriasis vulgaris, and 31 literatures whose relevant research information is incomplete and can't be 
Table 1 The basic characteristics of the included articles

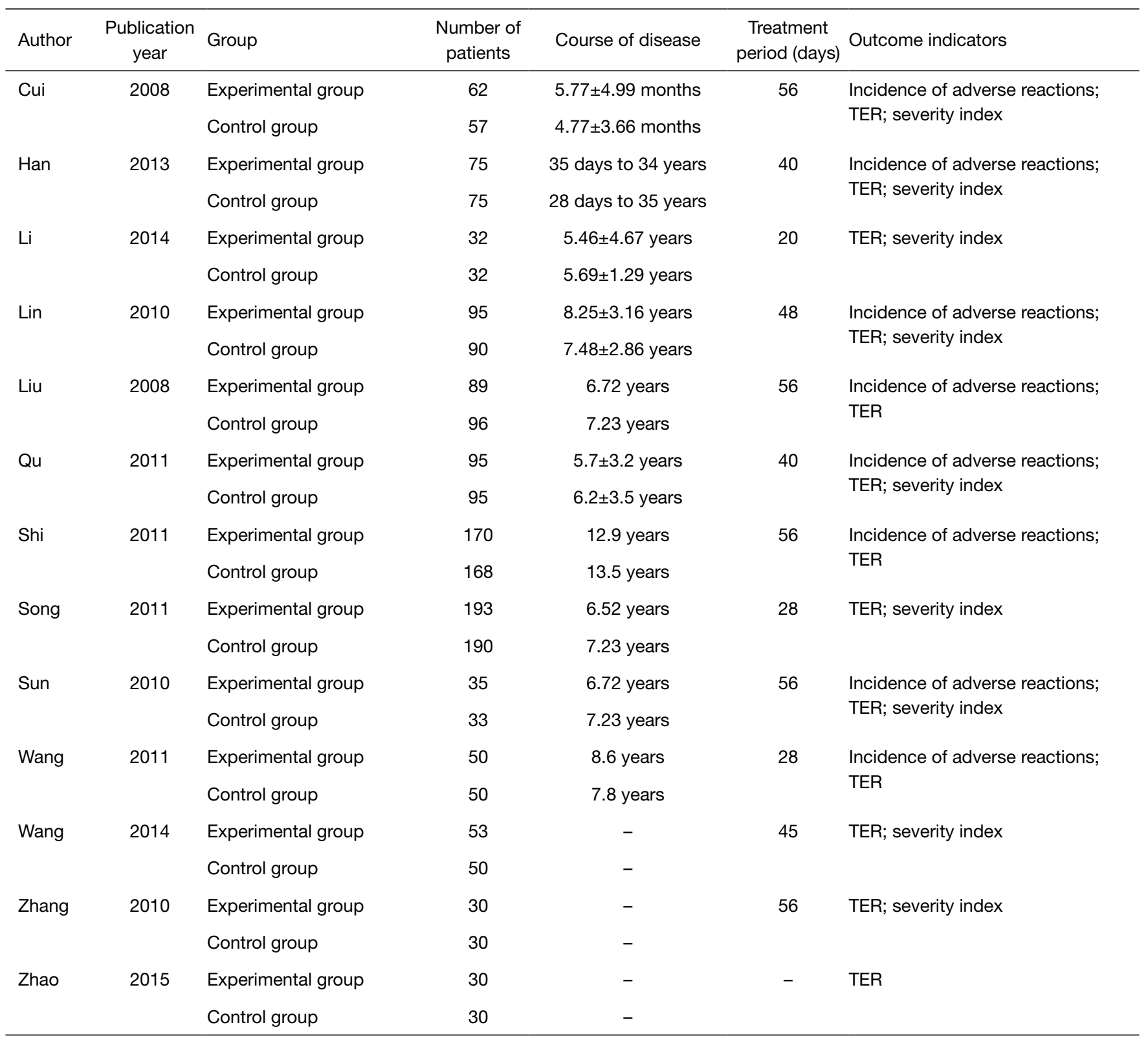

TER, total effective rate.

extracted after contacting the author. The recommendations of the NICE for quality evaluation are shown in Figure 2. It was revealed that 5 articles had a score of 6-8 points (accounting for $38.46 \%$ ), 7 articles had score of 3-5 points (accounting for $53.85 \%$ ), and 1 article had a score of $0-2$ points (accounting for $7.69 \%$ ).

A total of 13 articles with an accumulated 2,014 patient cases met the inclusion criteria, among which the sample size ranged from 30 to 193 and the average course of disease was about 6 years. The sample size, course of disease, treatment period, and outcome indicators were described in detail in 13 articles. Table 1 shows the general information and data of the included articles.

\section{Risk bias assessment of the included articles}

The software RevMan 5.3 was used to map the evaluation results of multiple risk biases in the included articles. 


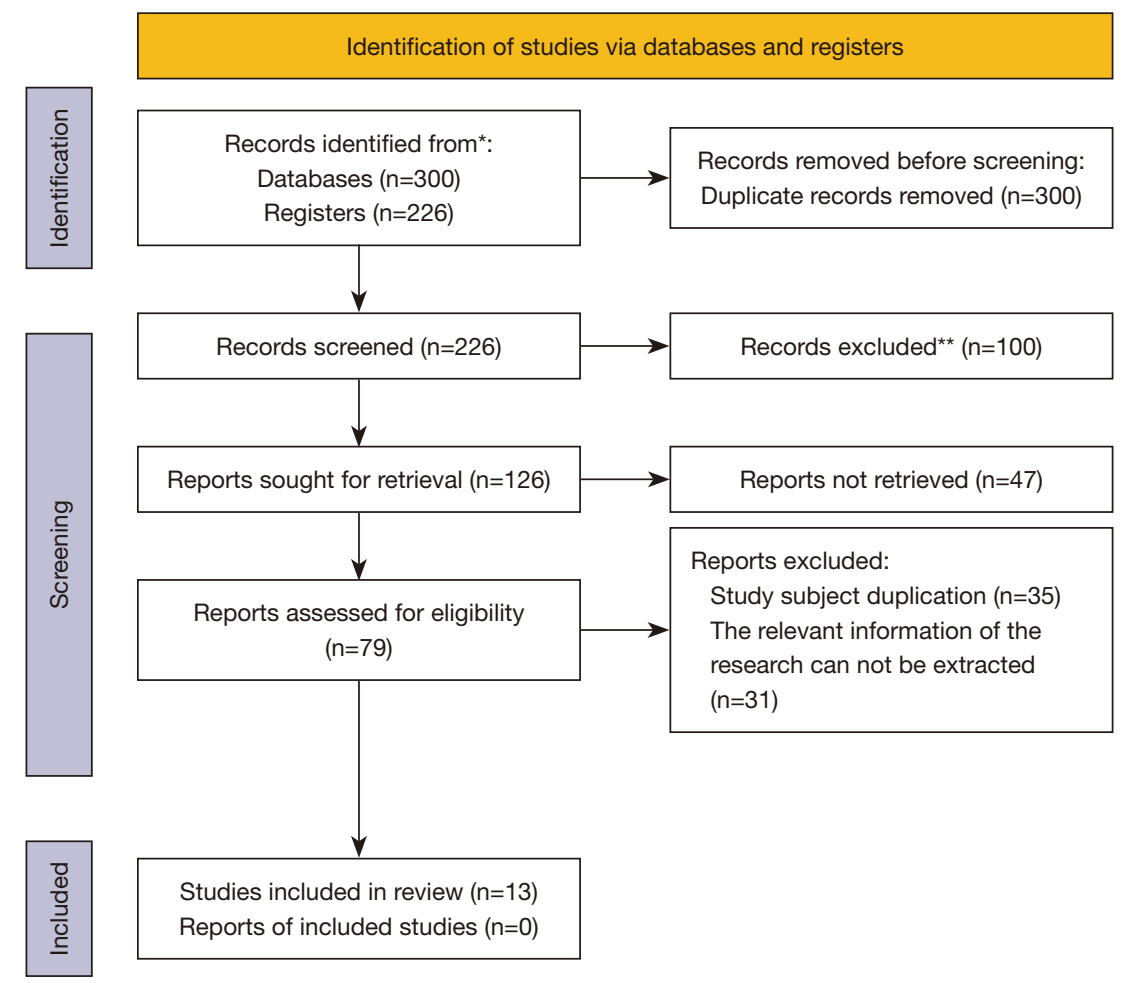

Figure 1 The flow chart for the article retrieval process. *, databases and registers; ${ }^{* *}$, duplicate literatures.

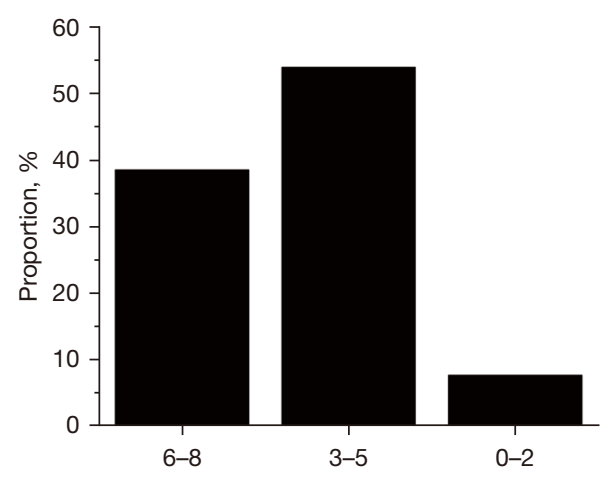

Figure 2 The recommendations of the NICE for quality evaluation. NICE, National Institute of Clinical Excellence.

The evaluation results were entered into the software to generate a bias risk evaluation chart. In this experimental study, there were a total of 2,005 cases in 13 articles, including 1,009 cases in the experimental group and 996 cases in the control group. In the 13 RCTs, 4 articles $(6,12,14,16)$ mentioned the randomization, accounting for $38.46 \%$. The results of the risk of deviation are shown in
Figures 3,4.

\section{Meta-analysis of TER}

A total of 13 articles (5-17) reported the TER, accounting for $100 \%$ of the total. Among them, there were 1,009 cases in the experimental group and 996 cases in the control group (2,005 cases in total). There was no heterogeneity in statistics $\left(\mathrm{P}=0.33, \mathrm{I}^{2}=11 \%\right)$, so a FEM was used. The results displayed in Figure 5 indicate that the meta-analysis revealed that the TER in the experimental group was higher than that in the control group, and the difference between the two groups was statistically significant $[\mathrm{n}=13$, odds ratio $(\mathrm{OR})=3.72, \mathrm{Z}=10.35, \mathrm{P}<0.00001,95 \% \mathrm{CI}: 2.90$ to 4.77$]$. The assessment of publication bias is shown in Figure 6. Among the 13 articles, the OR value of each study was set to the horizontal plane, and the standard error was set to the vertical plane. A funnel diagram of TER was drawn based on this, and the analysis results suggested that they were asymmetrically distributed in the two sides. The results indicated that the published articles may contain some 


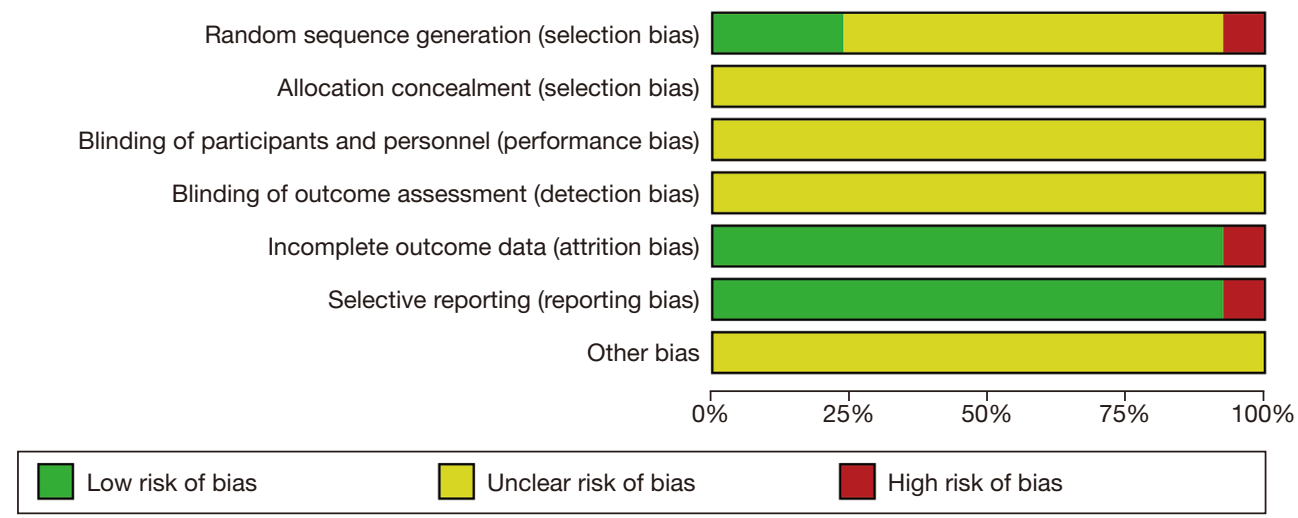

Figure 3 Assessment of risk bias of the included articles.

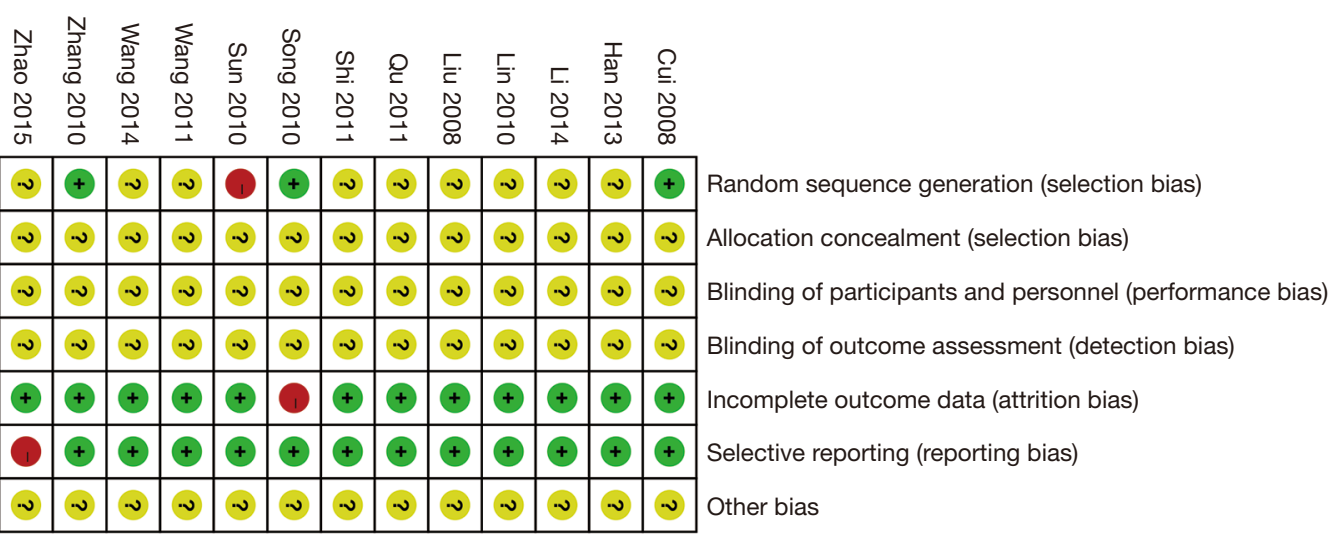

Figure 4 Multiple studies in the literature correspond to the multiple risk bias evaluation results.

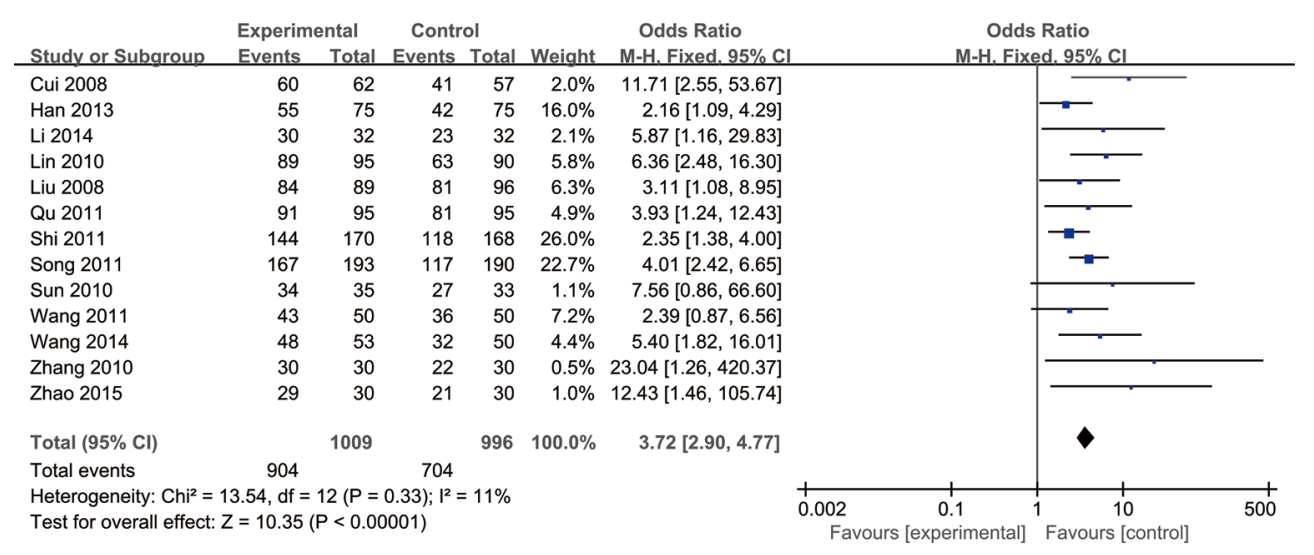

Figure 5 Forest map of TER. TER, total effective rate; CI, confidence interval. 


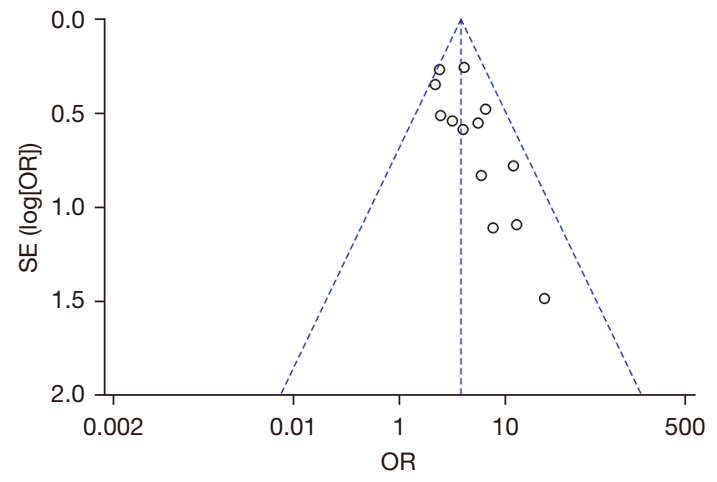

Figure 6 The funnel chart for bias of publication of the included articles in terms of TER. TER, total effective rate; SE, standard error; OR, odds ratio.

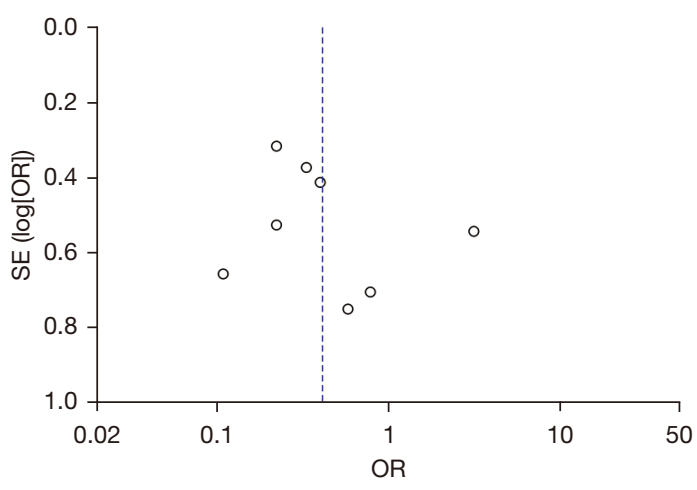

Figure 8 The funnel chart for bias of publication of the included articles in terms of incidence of adverse reactions. SE, standard error; OR, odds ratio.

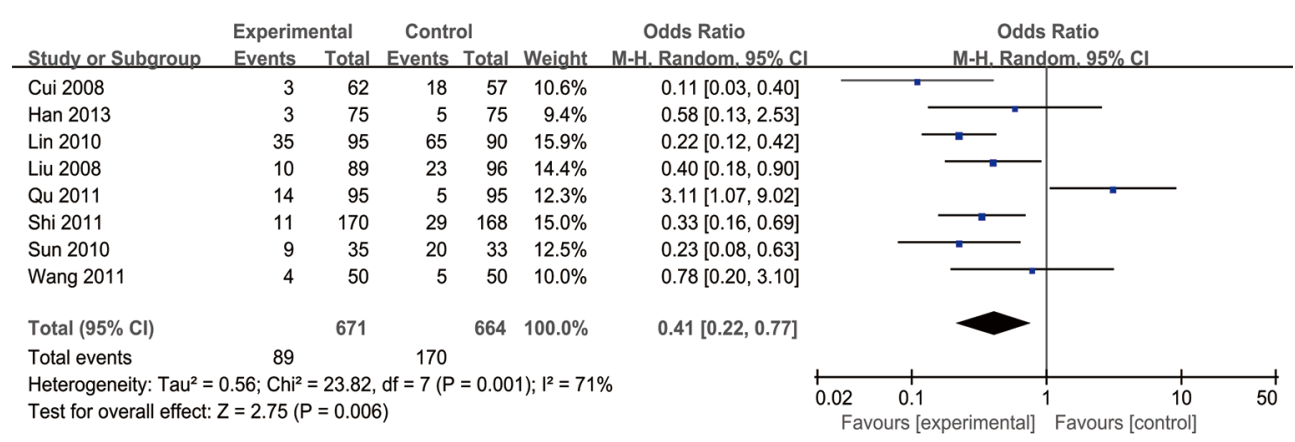

Figure 7 The forest map of incidence of adverse reactions. CI, confidence interval.

publication bias, which may be related to fewer publications of negative results.

\section{Meta-analysis on incidence of adverse reactions}

The incidence of adverse reactions was reported in 8 articles $(5,6,8-11,13,14)$, accounting for $61.53 \%$. Among them, there were 671 cases in the experimental group and 664 cases in the control group, with a total of 1,335 cases. There was no heterogeneity in statistics $\left(\mathrm{P}=0.001, \mathrm{I}^{2}=71 \%\right)$, so the REM was used. As shown in Figure 7, the results of meta-analysis revealed that the incidence of adverse reactions in the experimental group was lower in contrast to that in the control group, and the difference between the two groups was statistically great $(\mathrm{n}=8, \mathrm{OR}=0.41,95 \%$ CI: 0.22 to $0.77, \mathrm{Z}=2.75, \mathrm{P}=0.006$ ). The publication bias is shown in Figure 8 . In the 8 studies, the OR value was set to the horizontal plane and the standard error was set to the vertical plane, and a funnel chart of incidence of adverse reactions was drawn, which indicated that the distribution was asymmetric. The experimental results showed that the included articles may contain publication bias, which may be related to the low publication rate of articles with high incidence of adverse reactions.

\section{Meta-analysis on psoriasis area and severity index}

There were 9 articles $(5-8,10,12,13,15,16)$ which analyzed the psoriasis area and severity index, accounting for $69.23 \%$. There were 670 cased in the experimental group and 652 cases in the control group. The results showed that the psoriasis area and severity index scores in the experimental group were obviously lower than those of the control group, showing statistically great differences $[\mathrm{n}=9$, mean deviation $(\mathrm{MD})=-3.63,95 \% \mathrm{CI}:-5.38$ to $-1.88, \mathrm{Z}=4.07, \mathrm{P}<0.0001]$ (as illustrated in Figure 9). The publication for the psoriasis area and severity index are shown in Figure 10, which indicated that the distribution was asymmetric. The results 


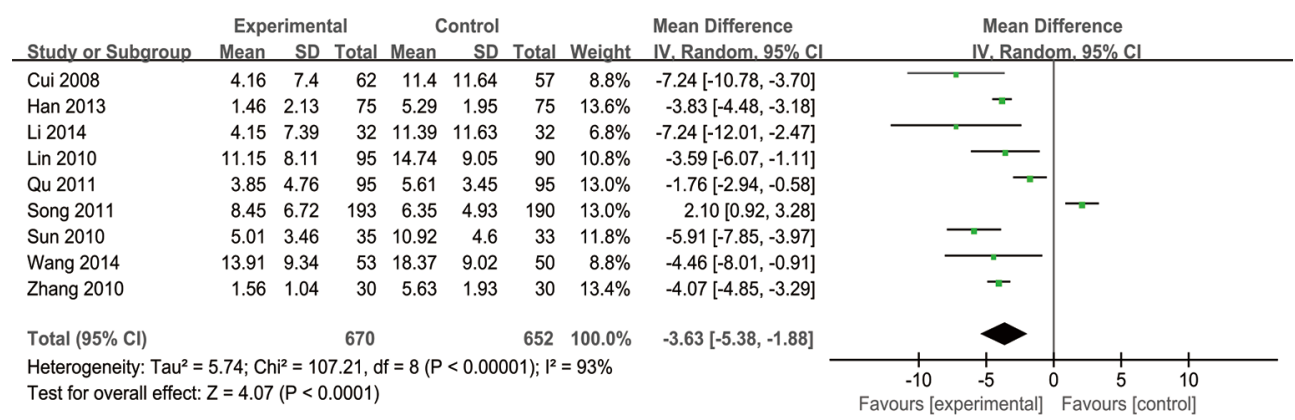

Figure 9 The forest map of psoriasis area and severity index. CI, confidence interval.

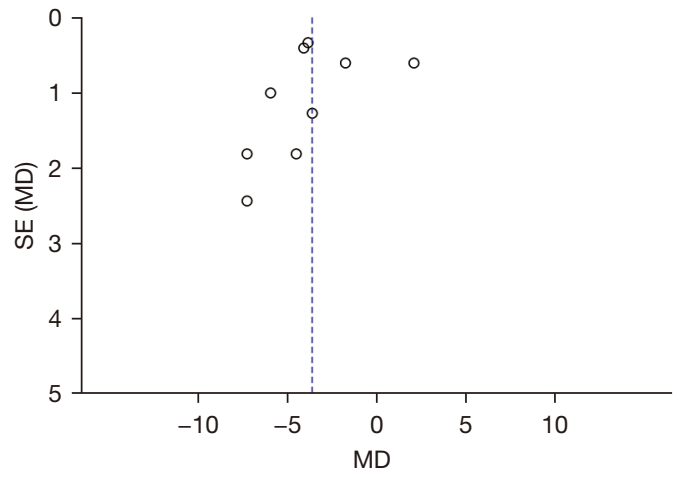

Figure 10 The funnel chart for bias of publication of the included articles in terms of psoriasis area and severity index. SE, standard error; MD, mean deviation.

indicated that the included articles may contain publication bias, which may be related to the relatively few articles with poor efficacy.

\section{Discussion}

At present, there is no specific treatment for psoriasis, but it is not an incurable disease. Proper symptomatic treatment can control the symptoms. Since the disease is a chronic relapsing disease, many patients need long-term treatment, and various therapies have certain adverse reactions, mainly including combination therapy, alternating therapy, sequential and intermittent therapy. The current treatment of psoriasis has the following points: (I) patients and doctors are pursuing radical cure, the purpose is not clear; (II) excessive treatment; (III) the treatment method is not standardized. As more and more psoriasis is studied (18), the number of people cured worldwide is also increasing (19). In particular, psoriasis vulgaris, due to its slow onset and easy to attack repeatedly, has attracted more attention from the medical community (20). Chinese medicinal bath enables the warm drugs to penetrate into the skin or acupoints and directly enter the meridians and collaterals, thus directly affecting the lesion sites. After the skin is washed by the soaking bath of the TCM medicinal bath, the pollutants such as exudation, crust, and scales can be removed, and the medicinal bath has the effects of moistening skin, relieving itching, clearing heat, cooling blood, and interpreting convergence; and the medicinal bath can expand the capillaries of patients, improve local microcirculation, promote metabolism, accelerate the repair of affected tissues, and promote the regression of skin lesions. In order to further improve the theoretical guidance on the treatment of psoriasis with herbal bath in China, 13 articles were included in the meta-analysis.

In 13 articles, the heterogeneity analysis of the TER of psoriasis vulgaris showed that there was no statistical heterogeneity, and the TER in the experimental group was higher than that in the control group, showing a statistically observable difference ( $\mathrm{P}<0.05,95 \%$ CI: 2.90 to 4.77), which indicated that TCM bath had a reliable therapeutic effect on psoriasis vulgaris. In these 13 articles, the results of funnel chart analysis revealed asymmetrical distribution, indicating that the published articles may contain some publication bias, which may be related to the lack of publications about negative results.

The incidence of adverse reactions was reported in 8 articles $(5,6,8-11,13,14)$, accounting for $61.53 \%$. The results revealed that there was no statistical heterogeneity, and the incidence of adverse reactions in the experimental group was lower in contrast to the incidence in control group, showing statistically obvious difference $(\mathrm{P}<0.05$, $95 \%$ CI: 0.22 to 0.77$)$. The funnel chart revealed that the distribution was asymmetric, so there was certain 
publication bias for the included articles, which may be related to the low publication rate of the articles with high incidence of adverse reactions. Using TCM bath can reduce the incidence of adverse reactions and is more helpful to the health of patients.

There were 9 articles reporting the psoriasis area and severity index, and the overall efficacy showed great differences between the two groups in the psoriasis area and severity index, with the score in experimental group lower in contrast to the control group $(\mathrm{P}<0.05,95 \%$ CI: -5.38 to -1.88$)$. The funnel chart showed asymmetry in the distribution, indicating that there may be publication bias in the included articles, which may be related to the relatively few articles with poor efficacy.

Among the 13 RCTs, 4 articles $(6,12,14,16)$ mentioned the randomization, accounting for $38.46 \%$. In this study, the clinical efficacy of TCM bath was systematically reviewed, and the ultraviolet radiation was combined to treat psoriasis. The objective of this study was to clarify the clinical treatment of psoriasis using TCM bathing. The selected cases and clinical efficacy were analyzed from TER, incidence of adverse reactions, psoriasis area, and severity index. A meta-analysis of the above indicators of psoriasis vulgaris was performed, based on which the funnel charts were drawn, which showed that the circles in some articles were not symmetrical to the midline, so the publication bias was visible. This may be related to the lack of publication of poorly effective articles. The conclusions obtained were relatively credible, and the quality level of the meta-analysis included in this study was in the upper-middle class.

\section{Conclusions}

In this study, a compound logic search method was used to retrieve 13 articles of TCM bathing in treatment of psoriasis vulgaris, and meta-analysis was adopted to evaluate the efficacy of this treatment method. The meta-analysis treatment based on TER, incidence of adverse reactions, and psoriasis area and severity index showed that most patients were cured effectively and the incidence of adverse reactions under the TCM bath was lower. The use of TCM bathing helped patients with psoriasis vulgaris to recover their health. The limitation of this study was that it mainly divided the participants according to their received treatment methods. The age, gender, and other diseases of the participants may have impacted on the experimental results, and the results obtained were not exact. The source of the sample had not been analyzed, and it may be affected by the equipment and subjective factors of the doctor. In summary, TCM bath treatment can be considered in the clinical treatment of psoriasis vulgaris.

\section{Acknowledgments}

Funding: None.

\section{Footnote}

Reporting Checklist: The authors have completed the PRISMA reporting checklist. Available at https://dx.doi. org/10.21037/apm-21-2386

Conflicts of Interest: All authors have completed the ICMJE uniform disclosure form (available at https://dx.doi. org/10.21037/apm-21-2386). The authors have no conflicts of interest to declare.

Ethical Statement: The authors are accountable for all aspects of the work in ensuring that questions related to the accuracy or integrity of any part of the work are appropriately investigated and resolved.

Open Access Statement: This is an Open Access article distributed in accordance with the Creative Commons Attribution-NonCommercial-NoDerivs 4.0 International License (CC BY-NC-ND 4.0), which permits the noncommercial replication and distribution of the article with the strict proviso that no changes or edits are made and the original work is properly cited (including links to both the formal publication through the relevant DOI and the license). See: https://creativecommons.org/licenses/by-nc-nd/4.0/.

\section{References}

1. Parker S, Zhang CS, Yu JJ, et al. Oral Chinese herbal medicine versus placebo for psoriasis vulgaris: a systematic review. J Dermatolog Treat 2017;28:21-31.

2. Xiang $\mathrm{Y}, \mathrm{Wu} \mathrm{X}, \mathrm{Lu} \mathrm{C}$, et al. An overview of acupuncture for psoriasis vulgaris, 2009-2014. J Dermatolog Treat 2017;28:221-8.

3. Yu JJ, Zhang CS, Coyle ME, et al. Compound glycyrrhizin plus conventional therapy for psoriasis vulgaris: a systematic review and meta-analysis of randomized controlled trials. Curr Med Res Opin 2017;33:279-87.

4. Guo X, Lang N, Li G, et al. Effectiveness of fleshmoistening paste in psoriasis vulgaris patients with symptom 
pattern of blood stasis: a randomized and parallel-controlled trial. J Tradit Chin Med 2020;40:1018-25.

5. Cui BN, Sun YX, Liu WL, et al. Clinical efficacy of narrow band ultraviolet bin combined with Yuyin recipe in treating psoriasis vulgaris. Chinese Journal of Integrated Traditional and Western Medicine 2008;28:355-7.

6. Han L, Zhao XM, Zhang H, et al. Curative effect of traditional Chinese medicine bath combined with narrowband UVB in the treatment of Psoriasis Vulgaris. Journal of Guiyang College of Traditional Chinese Medicine 2013;35:149-50.

7. Li N. Effect of Yuyin Recipe medicated bath combined with NB-UVB in the treatment of psoriasis vulgaris. China Health Industry 2014;(20):190-1.

8. Lin GS, Wang HY, Luo DF, et al. Clinical observation of traditional Chinese medical herbs bath combined with narrow-bind ultraviolet $B$ for the treatment of psoriasis vulgaris. Acta Universitatis Medicinalis Anhui 2010;45:404-6.

9. Liu HX, Gu Y, Zhang CH, et al. The observed of clinical curative effect of traditional Chinese medicine bath combined with narrow spectrum UVB radiation in the treatment of psoriasis vulgaris. In: Shijiazhuang: The Fifth Annual Conference of Dermatology Branch of Chinese Society of Chinese Medicine, 2008:176-8.

10. Qu YY, Yilizhafi A, Huang HP. Observation on therapeutic effect of pine tar baths combined with narrow-band ultraviolet $B$ in the treatment of psoriasis vulgade. Xinjiang Medical Journal 2011;41:28-30.

11. Shi XL, Pan YM, Ma HY, et al. Treatment of psoriasis vulgaris by NB-UVB combined with traditional Chinese materia medica bath: a clinical observation. Chinese Journal of Laser Medicine \& Surgery 2011;20:314-7.

12. Song LJ, Hu XH, Gao TW, et al. Curative effect of triple therapy in the treatment of psoriasis vulgaris. Chinese Journal of Dermatology 2011;44:60-1.

Cite this article as: Lei H, Chen M, Zhang N, Guo X, Yuan $\mathrm{X}$, Tang L, Ying C, Xie J. A systematic review and meta-analysis on the efficacy and safety of traditional Chinese medicine bath in the treatment of psoriasis vulgaris. Ann Palliat Med 2021;10(10):10674-10683. doi: 10.21037/apm-21-2386
13. Sun ZJ, Mi XL, Shi WJ, et al. Observation on the clinical curative effect of traditional Chinese medicine bath combined with narrow-band ultraviolet radiation in the treatment of psoriasis vulgaris. Contemporary Medicine 2010;16:152-3.

14. Wang ZX, Wang HJ, Yu ZH, et al. effect of the traditional Chinese medicine bath combined with NB-UVB in the treatment of psoriasis vulgaris. Journal of Henan University (Medical Science) 2011;(3):226-7.

15. Wang YH, Gao CR. Curative effect observation and nursing of NB-UVB combined with traditional Chinese medicine bath in the treatment of psoriasis vulgaris. Continuing Medical Education 2014;(12):84-5.

16. Zhang YS, Wei LP. Efficacy of NB-UVB combined with traditional Chinese medicine in the treatment of psoriasis vulgaris. China Journal of Leprosy And Skin Diseases 2010;26:815.

17. Zhao D, Huang JW, Huang RL. The clinical observation about Qinpi medicine bath joint NB-UVB in treating epidermis of psoriasis vulgaris. Clinical Journal of Traditional Chinese Medicine 2015;27:695-7.

18. Li T, Zhou D, Xu X, et al. Effect of traditional Chinese medicine plus narrow-band medium-wave ultraviolet $\mathrm{B}$ radiation on moderate-to-severe psoriasis vulgaris in a case series. J Tradit Chin Med 2019;39:692-9.

19. Coyle ME, Yu JJ, Zhang AL, et al. Patient experiences of using Chinese herbal medicine for psoriasis vulgaris and chronic urticaria: a qualitative study. J Dermatolog Treat 2020;31:352-8.

20. Chen X, Zhang R, Duan X, et al. Effectiveness of Xiaoyin Jiedu granules in the treatment of psoriasis vulgaris in patients with blood-heat symptom patterns in terms of Traditional Chinese Medicine. J Tradit Chin Med 2020;40:863-9.

(English Language Editor: J. Jones) 